\title{
Prostaglandin D synthase isoforms from cerebrospinal fluid vary with brain pathology
}

\author{
Michael G. Harrington ${ }^{\mathrm{a}, *}$, Alfred N. Fonteh ${ }^{\mathrm{a}}$, Roger G. Biringer ${ }^{\mathrm{b}}$, Andreas F. R. Hühmer ${ }^{\mathrm{b}}$ and \\ Robert P. Cowan ${ }^{\mathrm{a}}$ \\ ${ }^{a}$ Molecular Neurology Program, Huntington Medical Research Institutes, 99 North El Molino Avenue, Pasadena, \\ CA 91101, USA
}

Tel.: + 16267954343

${ }^{\mathrm{b}}$ Thermo Electron, 355 River Oaks Parkway, San Jose, CA 95134, USA

Tel.: + 14089656285

\begin{abstract}
Glutathione independent prostaglandin D synthase (Swissprot P41222, PTGDS) has been identified in human cerebrospinal fluid and some changes in PTGDS in relation to disease have been reported. However, little is known of the extent that PTGDS isoforms fluctuate across a large range of congenital and acquired diseases. The purpose of this study was to examine changes in PTGDS isoforms in such a population. Spinal fluid from 22 healthy study participants (normal controls) with no classifiable neurological or psychiatric diagnosis was obtained and PTGDS isoforms were identified by specific immunostaining and mass spectrometry after denaturing 2D gel electrophoresis. The PTGDS isoforms in controls consisted of five charge isoforms that were always present and a small number of occasional, low abundance isoforms. A qualitative survey of 98 different people with a wide range of congenital and acquired diseases revealed striking changes. Loss of the control isoforms occurred in congenital malformations of the nervous system. Gain of additional isoforms occurred in some degenerative, most demyelinating and vasculitic diseases, as well as in Creutzfeldt-Jakob disease. A retrospective analysis of published data that quantified relative amounts of PTGDS in multiple sclerosis, schizophrenia and Parkinson's disease compared to controls revealed significant dysregulation. It is concluded that qualitative and quantitative fluctuations of cerebrospinal fluid PTGDS isoforms reflect both major and subtle brain pathophysiology.
\end{abstract}

\section{Introduction}

The polyunsaturated fatty acid arachidonic acid (20:4, n-6) is oxygenated and reduced in the two-step, cyclooxygenase reaction to generate prostaglandin $\mathrm{G}_{2}$ $\left(\mathrm{PGG}_{2}\right)$ or prostaglandin $\mathrm{H}_{2}\left(\mathrm{PGH}_{2}\right)$, which in turn is converted to generate signaling molecules by an array of $\mathrm{PGH}_{2}$ metabolizing enzymes. One such enzyme catalyzes the conversion of $\mathrm{PGH}_{2}$ to prostaglandin $\mathrm{D}_{2}$ $\left(\mathrm{PGD}_{2}\right)$, a prostaglandin involved in pain, sleep, and smooth muscle contraction/relaxation, and a potent inhibitor of platelet aggregation [16,35]. This enzyme is glutathione independent prostaglandin D synthase (Swissprot P41222, PTGDS, also known as PGDS). PT-

\footnotetext{
*Corresponding author. E-mail: mghworks@hmri.org.
}

GDS has been identified in cerebrospinal fluid (CSF) [9, $11,20,25,34,39]$ and its isoforms have been reported as altered in neurological diseases [11,17,18,24,26-28].

The cause of these changes is not known, but altered PTGDS may indicate the source of cell pathophysiology: PTGDS is normally produced by meningeal and glial cells $[6,32,36]$. Consequences of altered PTGDS are also not known, but can be predicted to include variations in prostaglandins that may impact brain function because of their many cellular activities.

Our aim was to examine PTGDS and its many isoforms [11] in human cerebrospinal fluid (CSF) and determine, qualitatively, the range of variation in different diseases. To accomplish this, we surveyed PTGDS in CSF from patients with various congenital and acquired neurological diseases as well as from welldefined controls. Our prediction was that disorders 
that affected the cellular source of PTGDS or its downstream prostaglandin products would correlate with discernable deviations of this protein. We defined a profile of PTGDS isoforms from controls that was invariant and, when compared to this baseline, we found gains or losses of PTGDS isoforms in many diseases of the nervous system.

\section{Methods}

\section{1. $C S F$}

Samples from a total of 120 different persons were obtained from four sources, based on nationally accepted clinical diagnostic criteria: A) samples from 42 persons with different congenital or acquired diseases were obtained from the CSF bank at Duke University department of neurology $[1,2]$, under the direction of R.E.A.: alobar holoprosencephaly, schizencephaly with ventriculoperitoneal shunt, Arnold-Chiari malformation, myelomeningocele, hydranencephaly, neurometabolic disorder (negative biochemistry), immunodeficiency and metabolic disorder, glutaric aciduria Type 1, propionic academia, methyl malonic aciduria, HMG CoA lyase deficiency, Dandy Walker syndrome, prematurity with intraventricular hemorrhage, Lowe's syndrome, Pelizaeus-Merzbacher disease, Wilson's disease, Leigh's disease, Moyamoya with Down's syndrome, congenital myopathy, tuberosclerosis, Rett's syndrome, Kawasaki syndrome, fascio-scapulohumeral dystrophy, Down's syndrom with argininosuccinic aciduria, X-linked agammaglobulinemia, systemic lupus cerebritis, Niemann-Pick disease with dementia, schizophrenia (X2), Churg-Strauss syndrome, Wegener's granulomatosis, neurosyphilis, Alzheimer's, subdural hematoma, subarachnoid hemorrhage, normal pressure hydrocephalus, amyotrophic lateral sclerosis with dementia, multiple sclerosis, cerebral sarcoidosis, subacute cerebellar degeneration, B12 deficiency, and pneumococcal meningitis; B) three patients with MPTP-induced parkinsonism, from R.S. Burns, Springfield, Illinois; C) samples from 10 patients with suspected Creutzfeldt-Jakob disease referred to MGH, confirmed with a positive 14-3-3 $\gamma$ test [22], and 7 of these were pathologically verified at later autopsy; D) samples of 24 study participants with Alzheimer's disease, 9 with Parkinson's disease, 8 with multiple sclerosis, 2 with schizophrenia, and 22 with no classifiable psychiatric or neurological disorder after extensive evaluation ("normal" controls) were collected by
RPC and MGH as part of an ongoing ethically approved study at HMRI. All samples were collected by routine lumbar puncture, and stored frozen in aliquots at $-70^{\circ} \mathrm{C}$ before assay.

\subsection{Protein assay}

Concentrations of protein in CSF were determined using a micro titer plate-based Coomassie protein assay using human serum albumin (Sigma), $0-100 \mu \mathrm{g} / \mathrm{ml}$ as a standard [40]. Briefly, $5 \mu \mathrm{l}$ of diluted CSF (10 X and $100 \mathrm{X}$ ) was added to a 96 well microtiter plate in triplicate. Coomassie dye (BioRad, Hercules, CA) was diluted (5 X) and $200 \mu \mathrm{l}$ added to each well. After $5 \mathrm{~min}$, the OD at $595 \mathrm{~nm}$ was obtained using a microplate reader (Molecular Devices, Sunnyvale, CA) and protein concentrations in each sample were computed using Softmax software from Molecular Devices.

\subsection{Denaturing $2 D E$}

CSF proteins were precipitated by ethanol $(9 \mathrm{~mL}$ : $1 \mathrm{~mL}$ ethanol:CSF) and re-solubilized in a solution of $\beta 2$-mercaptoethanol, Triton X100, $8 \mathrm{M}$ urea, CHAPS and carrier ampholyte solution of $\mathrm{pH} 3-10$, and heated to $95^{\circ} \mathrm{C}$ for five minutes. Based on the total protein assay, $30 \mu \mathrm{g}$ of volume-reduced, denatured CSF protein per sample was applied to $16 \mathrm{~cm}$, carrier ampholyte gels for isoelectric focusing [12], followed by SDS PAGE on $14 \% \mathrm{~T}$ gels $(16 \mathrm{~cm}$ by $20 \mathrm{~cm})$, with detection by silver diamine staining [19]. Charge and mass standards are determined for the $x$ and $y$ axes respectively by $\mathrm{pI}$ calibration from published maps and comigration of molecular weight markers [38]. Approximately 1250 protein species were detected per sample. Immunodetection of PTGDS was achieved on identically run 2DE gels that were blotted to PVDF membrane for immunostaining. A rabbit polyclonal primary antibody specific for PTGDS [11] was employed, followed by secondary, horseradish peroxidase conjugated anti-rabbit anti-IgG reagent (Sigma), and these were visualized on the blot either with chromogen (chloronaphthol or diaminobenzidine) or by ECL chemiluminescence (Amersham Bioscience) and Kodak XAR5 film exposure. The constitutive isoforms of PTGDS from the control samples were defined and qualitative changes in samples from different congenital and acquired diseases were evaluated. Changes were noted if either a loss or gain of immunoreactive proteins were observed for the samples from disease-affected partici- 


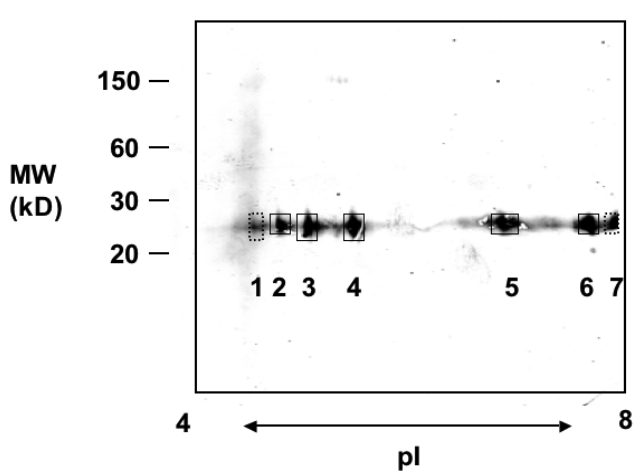

(A)

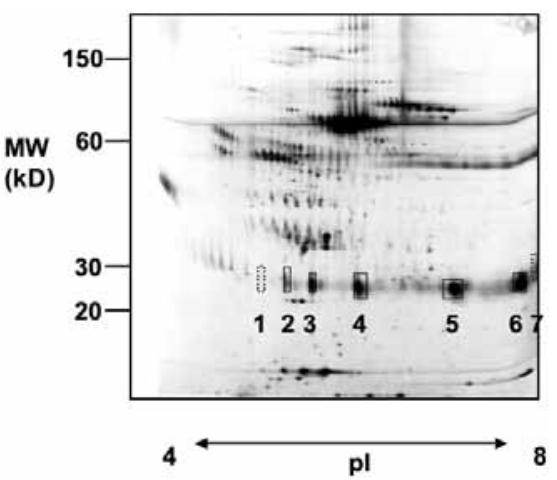

(B)

Fig. 1. (A) is a western blot of CSF after 2D gel separation as described in the Methods, from a study participant control with no classifiable psychiatric or neurologic disorder. The immunostain was specific for PTGDS, based on a synthetic peptide-derived antibody against the PTGDS sequence EAQVSVQPNFQQD. Molecular weight markers and pI distribution are noted on the $y$ and $x$ axis respectively. The invariant isoforms \#2-6 are indicated in continuous lined rectangles and the variant, faint isoform \#1 and variant "at-the-edge" basic \#7 are indicated at the sides, in broken lined rectangles. (B) is the image of the silver stained total CSF protein from the same sample. PTGDS isoforms are identified in the same manner as in $1 \mathrm{~A}$.

pants when compared with spot patterns and intensities from control gels.

Primary structural identification of proteins was completed using a scaled-up version of the $2 \mathrm{D}$ gel protocol described in the previous section. Briefly, 2-4 fold more total protein was separated on a $2 \mathrm{D}$ gel, directly stained with Sypro Ruby, and visualized using the Dark Reader (Claire Biochemicals) blue light box. Spots for proteins of interest were excised with a scalpel and subsequently prepared for mass spectrometry analysis. An aqueous solvent-based methodology for production and subsequent extraction of trypsin-derived peptides from gel plugs, was used [7]. Tryptic peptides were analyzed with an ion trap mass spectrometer (LCQ Deca, Thermo Electron) equipped with a nanoflow solvent delivery system and polymeric reversed-phase peptide trap (PepFinder Kit, Thermo Electron) to identify PTGDS isoforms. Peptides were identified from the mass spectra using Bioworks 3.1 (Thermo Electron), which correlates the experimental tandem mass spectra against theoretical tandem mass spectra from human protein via the Sequest algorithm [37]. Protein information was derived from the SWISS Prot database (Release 39) obtained from the Swiss Institute of Bioinformatics and contained 7459 human protein entries. Confidence in correct identifications by Bioworks was based on several factors: (repeated MS/MS analysis of the sample, repeated MS/MS of peptides within one run of the sample, MS/MS spectra quality, and a Bioworks Xcor and $\delta$ Cn greater than fairly stringent criteria [33]).

\section{Results}

\subsection{PTGDS identification}

Figure 1 illustrates the identification of the typical isoforms of PTGDS from a control CSF sample after immuno (1A) or silver (1B) staining. There were five isoforms that were invariably present in all controls, labeled \#'s 2-6. There was an additional fainter blob, \#1, that was not always present, presumably related to its low abundance, and a more basic isoform, \#7, at the basic edge of the $\mathrm{pH}$ range for IEF that was sometimes at the end. Another observation was the appearance in 3 of the 21 controls of very faint immunoreactive isoforms at a somewhat higher molecular weight (28$34 \mathrm{kD}$ ) in the basic region ( $\mathrm{pI} 5.8-7.5$ ), as indicated in Fig. 2. The same results were obtained with immobilized $\mathrm{pH}$ gradient IEF (data not shown). There was no observable qualitative change of these PTGDS immunoreactive spots with loading up to $200 \mu \mathrm{g}$ total protein.

Isoform \#'s 2-6 were consistently identified as PTGDS after excision, trypsin digestion, and LC/MS, performed as described in the Methods. Two peptides (APEAQVSVQPNFQQDK and AQGFTEDTIVFLPQTDK, both sequenced as doubly charged ions) were consistently derived from all five invariant normal isoforms. An example of the $\mathrm{MS}^{2}$ spectrum from isoform 2 is illustrated in Fig. 3. Notably, the only other proteins identified from these excised blobs were occasional immunoglobulin fragments, proteins known to have similar mass and charge, that were not immunoreactive with the antibody. 


\section{PTGDS in controls and patients with diseases of the nervous system}

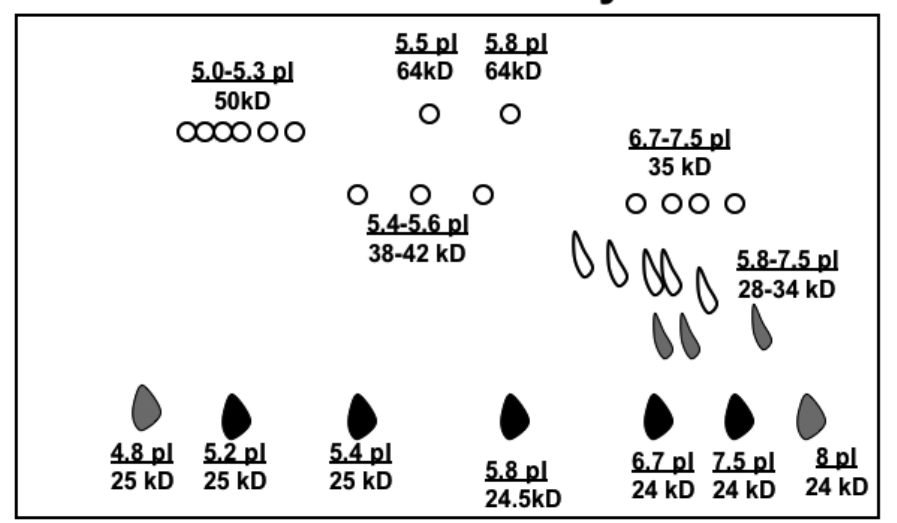

Proteins immunoreactive against anti-EAQVSVQPNFQQD

Fig. 2. This figure depicts the position of all PTGDS isoforms in CSF from control and different disease samples. The five black filled regions are invariant, while the gray filled regions are variable in controls, as described in the text. The higher molecular weight, white-filled regions are additional EAQVSVQPNFQQD immunoreactive isoforms that appear in select disease states, as described in the text. The numbers refer to $\mathrm{pI} / \mathrm{MW}(\mathrm{kD})$.

\subsection{Qualitative assessment of PTGDS}

In 22 healthy controls there were no qualitative differences for the 5 PTGDS isoforms across an age range of 19-97 yrs or between the genders, $10 \mathrm{~F}: 12 \mathrm{M}$, as reported in Table 1 . Two isoforms that were occasionally present were the acidic \#1 that was often too faint to be confidently identified and the \#7 that was at the variable basic edge of the isoelectric focusing. No sexual dimorphism was seen (data not shown).

In the disease groups, 28/98 had no gain or loss of the invariant 5 isoforms, as itemized in Table 1. However, there were dramatic changes in CSF from the other 70 persons (Table 1) and these are summarized in Fig. 2. All isoforms \# 2-6 (invariably present in controls) were absent from the CSF in hydranencephaly and one or more invariant forms were lost in schizencephaly, Arnold-Chiari malformation, myelomeningocele, Dandy-Walker syndrome, Wilson's disease, Leigh's disease and moyamoya disease with Down's syndrome. There were gains in additional PTGDS-immunoreactive spots in 64/98 samples studied, itemized in Table 1. An example of additional immunoreactive PTGDS species in the CSF from a participant with multiple sclerosis is illustrated in Fig. 4, compared to the same region 2D gel from a typical healthy control. In this analysis, many additional immunoreactive spots are visible in a "satellite" distribution around the normal isoforms.

\section{Discussion}

Identification of PTGDS in these experiments has relied on two independent techniques. The synthetic peptide-derived polyclonal antibody used throughout the study was highly specific, with no cross-reactivity to any other protein on the gels of the control samples, as seen in the western blot in Fig. 1(A) compared with the silver stained total proteins in Fig. 1(B). The characterization of isoform \#'s 2-6 by mass spectrometry from excised 2D gel blobs was of high confidence, as described in the methods, and as illustrated in Fig. 3.

This study is by no means comprehensive, but provides clear evidence of altered PTGDS isoforms in select disease types. Congenital malformations, two out of eleven people with mutations and one with severe hemorrhage led to loss of normal PTGDS isoforms. Additional PTGDS-immunoreactive isoforms appeared in most cases of multiple sclerosis, Creutzfeldt-Jakob disease and many neurodegenerative disorders such as Alzheimer's and Parkinson's diseases. It will be important to define the biochemical nature of the altered PTGDS-immunoreactive additional isoforms.

We know from our work and others that the variants of PTGDS are heavily glycosylated and all shift to a number of lower molecular weight basic forms after $\mathrm{N}$ - and O-linked deglycosylation [10,11,21]. It is also known that altered glycosyl transferases can lead 


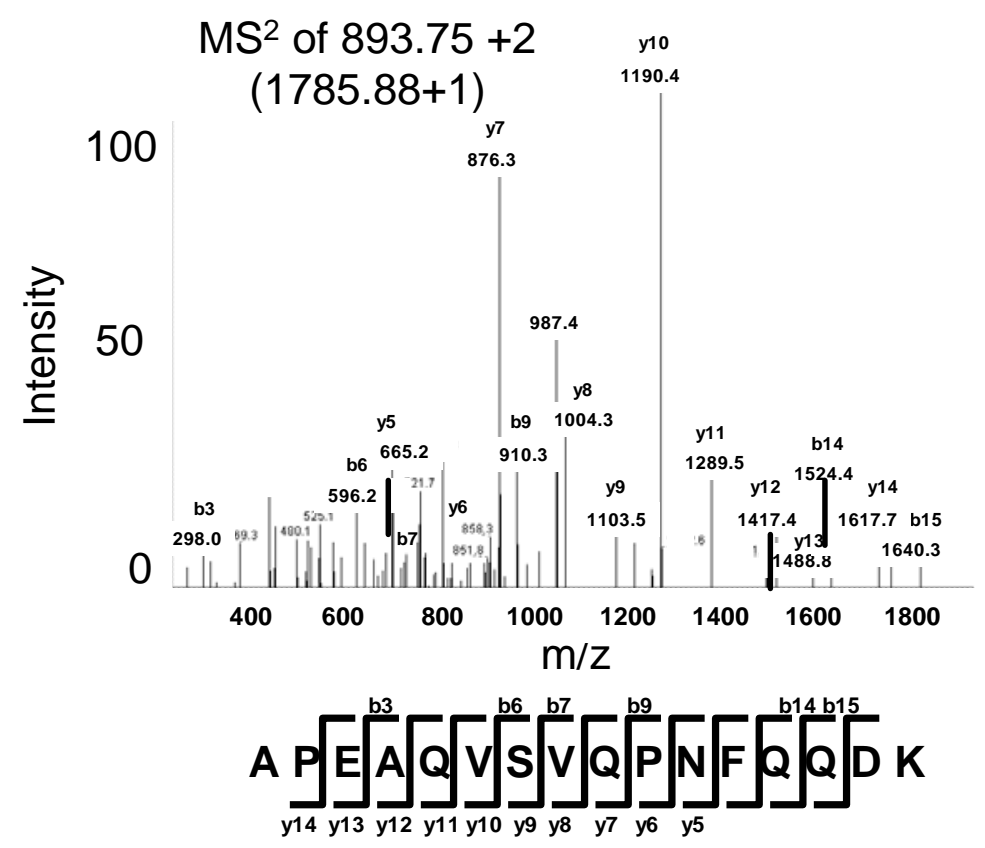

Fig. 3. The $\mathrm{MS}^{2}$ spectrum, with annotation, is presented for the doubly charged peptide APEAQVSVQPNFQQDK that was eluted from a gel plug of PTGDS spot \#3, and processed as described in the methods. The $y^{5-14}$ ions are indicated, as are six of the $b$ ions.

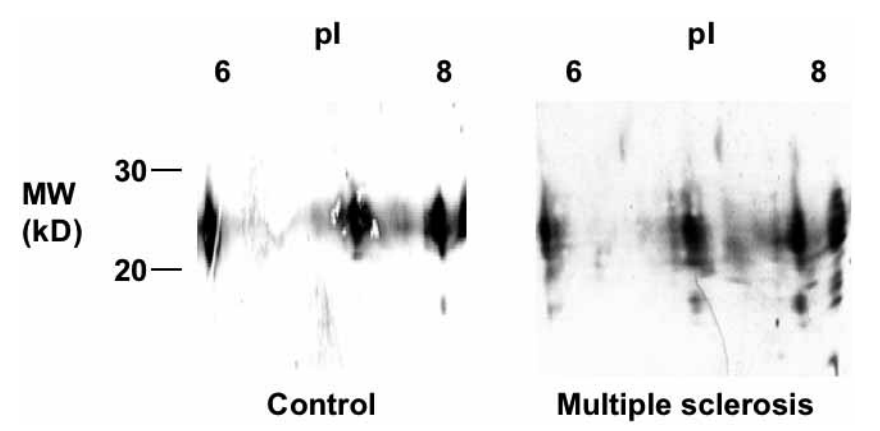

Fig. 4. The region of a western blot from a 2D gel of CSF from a control (no classifiable neurological or psychiatric disease) and a participant with multiple sclerosis, probed with anti-PTGDS as described in Methods. The additional immunoreactive isoforms around and above the normal PTGDS in the CSF from the MS participant are clearly visible. This result was typical for CSF from all CJD and MS participants, most cases of vasculitis and more than half of the neurodegenerative cases (see Table 1).

to variable glycoforms of PTGDS [8,23,31]. While the isoforms we observe may be differentially glycosylated, several other possibilities exist to explain the changes in IEF and gel mobility seen for normal and disease-derived isoforms. Genetic variants of PTGDS have been reported from brain $[3,29,30]$ and these may contribute to altered isoforms. Another possibility is that PTGDS is a small molecule carrier [5] and, although our 2D gels are denaturing, it is still possible that varied carrier states of retinoic acid, retinaldehyde, bilirubin or biliverdin could exist and lead to isoform variability. Casein kinase II phosphorylation of PT-
GDS has been reported [3,4] and thus isoforms may be altered by phosphorylation. In fact, it is not known whether any of the many possible post-translational modifications may be responsible for altered PTGDS isoforms in addition to the glycosylation. Further structural identification of the additional immunoreactive spots found in the 64 samples from people with different diseases will be required to more substantially test whether they are PTGDS variants and, if so, to characterize the modifications.

Qualitative assessment of the 22 controls revealed the PTGDS isoforms to be remarkably consistent. This 
Table 1

Clinical data of study participants and their PTGDS correlates

Etiology*
Congenital malformation
Inborn error of metabolism and
other mutations
Infectious
Inflammatory
Degenerative
Hemorrhagic
Metabolic
Toxic
Nutritional
Unclassified
Controls (no classifiable disease)

$\begin{array}{cc}\begin{array}{c}\text { \# of cases and } \\ \text { gender ratio (F:M) }\end{array} & \text { Age range } \\ 7(4: 3) & 1 \mathrm{~d}-27 \mathrm{y} \\ & \\ 15(10: 5) & 1 \mathrm{~m}-38 \mathrm{y} \\ & \\ 12(6: 6) & 30-76 \mathrm{y} \\ 14(10: 4) & 4-65 \mathrm{y} \\ 36(18: 18) & 37-91 \mathrm{y} \\ 3(1: 2) & 16 \mathrm{~d}-55 \mathrm{y} \\ 2(1: 1) & 7-8 \mathrm{y} \\ 3(1: 2) & 28-33 \mathrm{y} \\ 1(1: 0) & 67 \mathrm{y} \\ 5(1: 4) & 24-79 \mathrm{y} \\ 22(10: 12) & 19-97 \mathrm{y}\end{array}$

Normal

Loss of
Normal PTGDS
6 lose 1
1 loses all
2 lose 1

Gain of additional PTGDS immuno-reactive isoforms

0
12
2
1
6
1
2
3
1
0
22

$$
\begin{array}{r}
6 \\
1 \\
10 \\
13 \\
30 \\
1 \\
0 \\
0 \\
0 \\
5 \\
0
\end{array}
$$
30
${ }^{*}$ Congenital $=$ alobar holoprosencephaly, schizencephaly with ventriculoperitoneal shunt, Arnold-Chiari malformation, myelomeningocele, hydranencephaly, Dandy Walker syndrome, Lowe's syndrome; Inborn errors of metabolism/mutations = glutaric aciduria Type 1, propionic academia, methyl malonic aciduria, HMG CoA lyase deficiency, Pelizaeus-Merzbacher disease, Wilson's disease, Leigh's disease, congenital myopathy, tuberosclerosis, Rett's syndrome, fascio-scapulo-humeral dystrophy, Down's syndrome with argininosuccinic aciduria, X-linked agammaglobulinemia, Moyamoya with Down's syndrome, Niemann-Pick disease with dementia; Infectious = neurosyphilis, pneumococcal meningitis, Creutzfeldt-Jakob disease (10 cases); Inflammatory = Kawasaki syndrome, systemic lupus cerebritis, Churg-Strauss syndrome, Wegener's granulomatosis, multiple sclerosis (9 cases), cerebral sarcoidosis; Degenerative = Alzheimer's $(25$ cases), Parkinson's disease (9 cases), amyotrophic lateral sclerosis with dementia, subacute cerebellar degeneration; Hemorrhagic = prematurity with intraventricular hemorrhage, subdural hematoma, subarachnoid hemorrhage; Metabolic = neurometabolic disorder (negative biochemistry), immunodeficiency and metabolic disorder; Toxic $=$ MPTP-induced parkinsonism (3 cases); Nutritional = B12 deficiency; Unclassified = schizophrenia $(4$ cases $)$, normal pressure hydrocephalus.

Table 2

Relative changes in PTGDS protein quantity in CSF from patients with multiple sclerosis, Parkinson's disease, and schizophrenia compared to 100 control subjects [13-15]

$\begin{array}{lccc}\text { PTGDS } & \text { Multiple sclerosis } & \text { Parkinson's disease } & \text { Schizophrenia } \\ & n=22 & n=20 & n=54 \\ \text { pI 5.2, invariant \#3 } & \text { Not significant } & \text { Decreased } p<0.0005 & \text { Not significant } \\ \text { pI 5.4, invariant \#4 } & \text { Decreased } p<0.05 & \text { Decreased } p<0.005 & \text { Decreased } p<0.005 \\ \text { pI 6.7, invariant \#5 } & \text { Increased } p<0.01 & \text { Not significant } & \text { Not significant } \\ \text { Global PTGDS } & \text { Increased } & \text { No change } & \text { No change } \\ \text { Higher MW (30-45) } & \text { Yes, in all } & \text { No } & \text { Yes, in one third }\end{array}$

would argue against significant frequency of genetic polymorphism, although obviously a larger population will be necessary to fully evaluate this. This constancy of the control references provides a striking contrast to the samples where the isoforms that are normally present disappear, or when additional isoforms appear. As mentioned above, definitive structural identification of these added variants is needed, but the specificity of the immunoreactivity makes their PTGDS polypeptide backbone likely.

What may be the explanation for the changes in PTGDS forms? Loss of the five control isoforms likely represents a serious cellular defect in PTGDS regulation. It is interesting that the only conditions we have observed total or partial loss of the invariant five forms were in congenital malformations of the brain/meninges or other severe brain pathologies (Table 1). Since the sites of normal PTGDS synthesis are

the glial cells and meninges, perhaps this is not surprising in conditions that damage these cells. The PTGDS gene may still be expressed, but processed differently. Such loss of the normal $25 \mathrm{kD}$ isoforms has already been reported in extracts from many other tissues with different size and charge products compared to the normal CSF pattern [11]. To explain the major charge/mass shifts of immunoreactive PGTDS, one must invoke alternative splicing, translational processing or other post-translational modifications.

The cause of the gain of additional isoforms, such as illustrated in Fig. 4, will only be elucidated with definition of their structure. Changes in the regulation of glycosylation likely lead to the altered expressed forms, but there are many alternative possibilities, including phosphorylation, acylation, glycation or oxidation.

What consequences may result from the gain or loss of PTGDS isoforms? It is likely that many downstream 
lipid mediators will be affected when PTGDS is altered: prostaglandin fluctuations will change brain inflammation, immune response, aggregation, chemotaxis, vasodilation, ion channels, cell growth/differentiation, pain and sleep functions. Since these are recognized features in the disorders that have altered PTGDS forms, the altered prostaglandins may directly contribute to the disease manifestations. It seems worthwhile to continue to define these PTGDS changes, as it is only with such a molecular definition of pathology in specific disease conditions that corrective therapy can be rationally explored.

This study was qualitative. However, we have published quantitative data from diseases that we studied prior to characterizing PTGDS. We quantified isoforms that we now know as PTGDS, from 54 patients with schizophrenia [13], 20 with idiopathic Parkinson's disease [14], and 22 with multiple sclerosis [15], compared to 100 controls. In those studies we reported differential up/down regulation of isoforms. We have extracted this data in the context of PTGDS from those references and summarized it in Table 2, where the isoforms previously described by $\mathrm{pI}$ and $\mathrm{MW}$ as $(5.2,25 \mathrm{kD}),(5.4$, $25 \mathrm{kD}),(6.7,24 \mathrm{kD})$ correspond to invariant PTGDS isoforms (\#3), (\#4), (\#5), respectively.

This quantitative measure of PTGDS dysregulation in disease further supports the qualitative data from this study. Combining the qualitative and quantitative data, there are multiple lines of evidence supporting the prediction that altered PTGDS reflects underlying meningeal/glial pathophysiology. The only losses of PTGDS occur in congenital malformations of the leptomeninges and massive structural brain damage. The appearances of additional PTGDS isoforms occur in diseases with the common feature of glial pathology and at locations known to be the main sources of PTGDS mRNA $[6,32,36]$, exemplified by multiple sclerosis. The significant quantitative changes in schizophrenia, multiple sclerosis and Parkinson's disease may be indicators of more subtle changes in glial pathology in these conditions. Further study of PTGDS should illuminate understanding of these brain disorders.

\section{Conclusions}

PTGDS, a heavily glycosylated protein, was found to have five isoforms in human CSF from all controls who had no classifiable psychiatric or neurological disease $(n=22)$. The same proteins were present in a wide variety of nervous system disorders, includ- ing many different inborn errors of metabolism, infections, neoplasms and degenerative disease. However, losses of these invariant PTGDS isoforms were observed in congenital malformations of the nervous system, and additional isoforms were detected in malformations, Creutzfeldt-Jakob disease, vasculitic, demyelinating and hemorrhagic disorders. Review of the quantitative data of three invariant PTGDS isoforms from prior published work reveals that statistically significant differential up/down regulation of PTGDS occurs in multiple sclerosis, Parkinson's disease and schizophrenia compared to controls [13-15].

Alterations in PTGDS represent pathology at the cellular level and will produce fluctuations in prostaglandins that correlate with symptoms in these diseases. Figure 2 provides an isoform map for PTGDS, these isoforms are candidate diagnostic biomarkers of disease and they may have predictive value. Further study of this protein, its isoforms, and the other enzymes and substrates of the cyclooxygenase pathway, along with clinical correlations, will likely add to our understanding of the pathophysiology of brain disorders.

\section{References}

[1] R.E. Albright, Jr., R.H. Christenson, R.L. Habig, T.P. Mears and K.A. Schneider, Cerebrospinal fluid (CSF) TRAP. A method to improve CSF laboratory efficiency, Am J Clin Pathol 90 (1988), 707-710.

[2] R.E. Albright, Jr., R.H. Christenson, S.C. Schold, Jr., K.A. Schneider, R.L. Habig, W.W. Johnston, M.P. Pickett, W.F. Kulik, Sr. and T.P. Mears, Establishment of a CSF bank, Neurology 38 (1988), 492-494.

[3] F. Angenstein, K. Buchner and S. Staak, Age-dependent differences in glutamate-induced phosphorylation systems in rat hippocampal slices, Hippocampus 9 (1999), 173-185.

[4] W.M. Armstead, Role of nitric oxide and cAMP in prostaglandin-induced pial arterial vasodilation, Am J Physiol 268 (1995), H1436-1440.

[5] C.T. Beuckmann, M. Aoyagi, I. Okazaki, T. Hiroike, H. Toh, O. Hayaishi and Y. Urade, Binding of biliverdin, bilirubin, and thyroid hormones to lipocalin-type prostaglandin D synthase, Biochemistry 38 (1999), 8006-8013.

[6] C.T. Beuckmann, M. Lazarus, D. Gerashchenko, A. Mizoguchi, S. Nomura, I. Mohri, A. Uesugi, T. Kaneko, N. Mizuno, O. Hayaishi and Y. Urade, Cellular localization of lipocalin-type prostaglandin D synthase (beta-trace) in the central nervous system of the adult rat, J Comp Neurol $\mathbf{4 2 8}$ (2000), 62-78.

[7] R.G. Biringer, M.G. Harrington, W. Stochaj, P. Bondarenko, A. Huhmer, H. Amato, G. Chu and S. Swedberg, Identification of proteins from Human cerebrospinal fluid by twodimensional electrophoresis and ion-trap mass spectrometry: Improved sample preparation and presentation methods, Abstract P112-S, At: http://abrf.org/Other/ABRFMeetings /ABRF2002/2002Abstracts.html, 2002. 
[8] E. Grabenhorst, A. Hoffmann, M. Nimtz, G. Zettlmeissl and H.S. Conradt, Construction of stable BHK-21 cells coexpressing human secretory glycoproteins and human Gal(beta 1-4)GlcNAc-R alpha 2,6-sialyltransferase alpha 2,6linked NeuAc is preferentially attached to the Gal(beta 14)GlcNAc(beta 1-2)Man(alpha 1-3)-branch of diantennary oligosaccharides from secreted recombinant beta-trace protein, Eur J Biochem 232 (1995), 718-725.

[9] D.E. Griffin, S.L. Wesselingh and J.C. McArthur, Elevated central nervous system prostaglandins in human immunodeficiency virus-associated dementia, Ann Neurol 35 (1994), 592-597.

[10] K. Hakansson, M.R. Emmett, A.G. Marshall, P. Davidsson and C.L. Nilsson, Structural analysis of 2D-gel-separated glycoproteins from human cerebrospinal fluid by tandem highresolution mass spectrometry, J Proteome Res 2 (2003), 581588.

[11] M.G. Harrington, R. Aebersold, B.M. Martin, C.R. Merril and L. Hood, Identification of a brain-specific human cerebrospinal fluid glycoprotein, beta-trace protein, Appl Theor Electrophor 3 (1993), 229-234.

[12] M.G. Harrington, D. Gudeman, T. Zewert, M. Yun and L. Hood, Analytical and micropreparative two-dimensional electrophoresis of proteins, Methods: A companion to Methods in Enzymology 3 (1991), 98-108.

[13] M.G. Harrington and C.R. Merril, Additional cerebrospinal fluid proteins found in schizophrenia and Creutzfeldt-Jakob disease, Psychopharmacol Bull 21 (1985), 361-364.

[14] M.G. Harrington and C.R. Merril, Two-dimensional electrophoresis and "ultrasensitive" silver staining of cerebrospinal fluid proteins in neurological diseases, Clin Chem 30 (1984), 1933-1937.

[15] M.G. Harrington, C.R. Merril, D. Goldman, X. Xu and D.E. McFarlin, Two-dimensional electrophoresis of cerebrospinal fluid proteins in multiple sclerosis and various neurological diseases, Electrophoresis 5 (1984), 236-245.

[16] O. Hayaishi and Y. Urade, Prostaglandin D2 in sleep-wake regulation: recent progress and perspectives, Neuroscientist $\mathbf{8}$ (2002), 12-15.

[17] A. Hiraoka, T. Arato, I. Tominaga, N. Eguchi, H. Oda and Y. Urade, Sodium dodecyl sulfate-capillary gel electrophoretic analysis of molecular mass microheterogeneity of beta-trace protein in cerebrospinal fluid from patients with central nervous system diseases, J Chromatogr A 802 (1998), 143-148.

[18] A. Hiraoka, K. Seiki, H. Oda, N. Eguchi, Y. Urade, I. Tominaga and $\mathrm{K}$. Baba, Charge microheterogeneity of the betatrace proteins (lipocalin-type prostaglandin D synthase) in the cerebrospinal fluid of patients with neurological disorders analyzed by capillary isoelectrofocusing, Electrophoresis $\mathbf{2 2}$ (2001), 3433-3437.

[19] D.F. Hochstrasser, M.G. Harrington, A.C. Hochstrasser, M.J. Miller and C.R. Merril, Methods for increasing the resolution of two-dimensional protein electrophoresis, Anal Biochem 173 (1988), 424-435.

[20] A. Hoffmann, H.S. Conradt, G. Gross, M. Nimtz, F. Lottspeich and U. Wurster, Purification and chemical characterization of beta-trace protein from human cerebrospinal fluid: its identification as prostaglandin D synthase, J Neurochem 61 (1993), $451-456$.

[21] A. Hoffmann, M. Nimtz, U. Wurster and H.S. Conradt, Carbohydrate structures of beta-trace protein from human cerebrospinal fluid: evidence for "brain-type" N-glycosylation, $J$ Neurochem 63 (1994), 2185-2196.
[22] G. Hsich, K. Kenney, C.J. Gibbs, K.H. Lee and M.G. Harrington, The 14-3-3 brain protein in cerebrospinal fluid as a marker for transmissible spongiform encephalopathies, $N$ Engl J Med 335 (1996), 924-930.

[23] T. Imbach, B. Schenk, E. Schollen, P. Burda, A. Stutz, S. Grunewald, N.M. Bailie, M.D. King, J. Jaeken, G. Matthijs, E.G. Berger, M. Aebi and T. Hennet, Deficiency of dolicholphosphate-mannose synthase-1 causes congenital disorder of glycosylation type Ie, J Clin Invest 105 (2000), 233-239.

[24] M. Kawashima, S.O. Suzuki, T. Yamashima, M. Fukui and T. Iwaki, Prostaglandin D synthase (beta-trace) in meningeal hemangiopericytoma, Mod Pathol 14 (2001), 197-201.

[25] A.P. Kuruvilla, G.M. Hochwald, J. Ghiso, E.M. Castano, M. Pizzolato and B. Frangione, Isolation and amino terminal sequence of beta-trace, a novel protein from human cerebrospinal fluid, Brain Res 565 (1991), 337-340.

[26] M. Mase, K. Yamada, A. Iwata, T. Matsumoto, K. Seiki, H. Oda and Y. Urade, Acute and transient increase of lipocalintype prostaglandin D synthase (beta-trace) level in cerebrospinal fluid of patients with aneurysmal subarachnoid hemorrhage, Neurosci Lett 270 (1999), 188-190.

[27] M. Mase, K. Yamada, N. Shimazu, K. Seiki, H. Oda, H. Nakau, T. Inui, W. Li, N. Eguchi and Y. Urade, Lipocalintype prostaglandin D synthase (beta-trace) in cerebrospinal fluid: a useful marker for the diagnosis of normal pressure hydrocephalus, Neurosci Res 47 (2003), 455-459.

[28] D.N. Melegos, M.S. Freedman and E.P. Diamandis, Prostaglandin D synthase concentration in cerebrospinal fluid and serum of patients with neurological disorders, Prostaglandins 54 (1997), 463-474.

[29] Y. Miwa, S. Takiuchi, K. Kamide, M. Yoshii, T. Horio, C. Tanaka, M. Banno, T. Miyata, T. Sasaguri and Y. Kawano, Identification of gene polymorphism in lipocalintype prostaglandin D synthase and its association with carotid atherosclerosis in Japanese hypertensive patients, Biochem Biophys Res Commun 322 (2004), 428-433.

[30] A. Nagata, Y. Suzuki, M. Igarashi, N. Eguchi, H. Toh, Y. Urade and O. Hayaishi, Human brain prostaglandin D synthase has been evolutionarily differentiated from lipophilic-ligand carrier proteins, Proc Natl Acad Sci USA 88 (1991), 40204024.

[31] S. Pohl, A. Hoffmann, A. Rudiger, M. Nimtz, J. Jaeken and H.S. Conradt, Hypoglycosylation of a brain glycoprotein (beta-trace protein) in CDG syndromes due to phosphomannomutase deficiency and $\mathrm{N}$-acetylglucosaminyl-transferase II deficiency, Glycobiology 7 (1997), 1077-1084.

[32] M. Taniike, I. Mohri, N. Eguchi, C.T. Beuckmann, K. Suzuki and Y. Urade, Perineuronal oligodendrocytes protect against neuronal apoptosis through the production of lipocalin-type prostaglandin D synthase in a genetic demyelinating model, $J$ Neurosci 22 (2002), 4885-4896.

[33] M.P. Washburn, R. Ulaszek, C. Deciu, D.M. Schieltz and J.R. Yates, 3rd, Analysis of quantitative proteomic data generated via multidimensional protein identification technology, Anal Chem 74 (2002), 1650-1657.

[34] K. Watanabe, Y. Urade, M. Mader, C. Murphy and O. Hayaishi, Identification of beta-trace as prostaglandin D synthase, Biochem Biophys Res Commun 203 (1994), 1110-1116.

[35] T. Watanabe, S. Narumiya, T. Shimizu and O. Hayaishi, Characterization of the biosynthetic pathway of prostaglandin D2 in human platelet-rich plasma, J Biol Chem 257 (1982), 1484714853.

[36] T. Yamashima, K. Sakuda, Y. Tohma, J. Yamashita, H. Oda, D. Irikura, N. Eguchi, C.T. Beuckmann, Y. Kanaoka, Y. Urade 
and O. Hayaishi, Prostaglandin D synthase (beta-trace) in human arachnoid and meningioma cells: roles as a cell marker or in cerebrospinal fluid absorption, tumorigenesis, and calcification process, J Neurosci 17 (1997), 2376-2382.

[37] J.R. Yates, 3rd., J.K. Eng and A.L. McCormack, Mining genomes: correlating tandem mass spectra of modified and unmodified peptides to sequences in nucleotide databases, Anal Chem 67 (1995), 3202-3210.

[38] M. Yun, W. Wu, L. Hood and M. Harrington, Human cere- brospinal fluid protein database: edition 1992, Electrophoresis 13 (1992), 1002-1013.

[39] M. Zahn, M. Mader, B. Schmidt, E. Bollensen and K. Felgenhauer, Purification and N-terminal sequence of beta-trace, a protein abundant in human cerebrospinal fluid, Neurosci Lett 154 (1993), 93-95.

[40] S.S.Zuo and P. Lundahl, A micro-Bradford membrane protein assay, Anal Biochem 284 (2000), 162-164. 


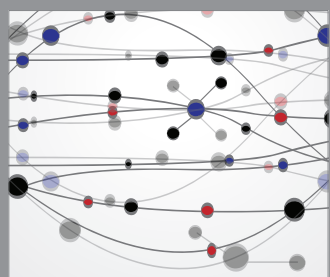

The Scientific World Journal
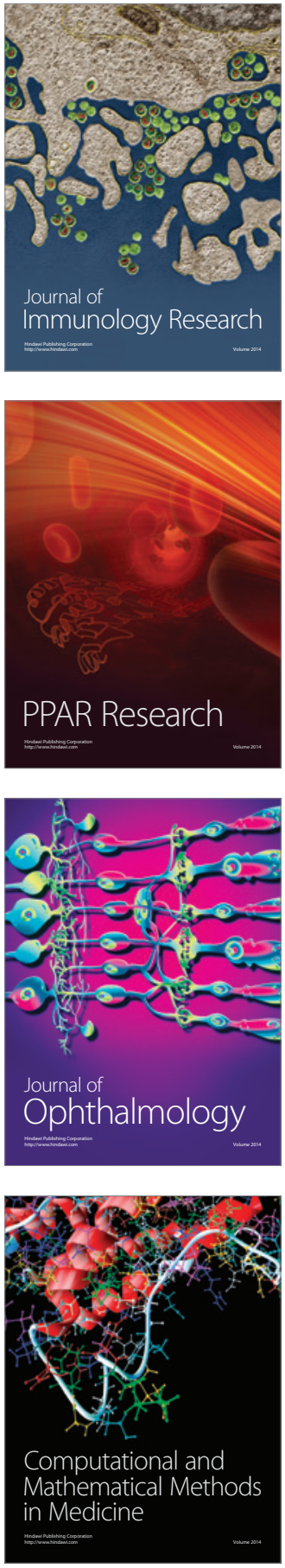

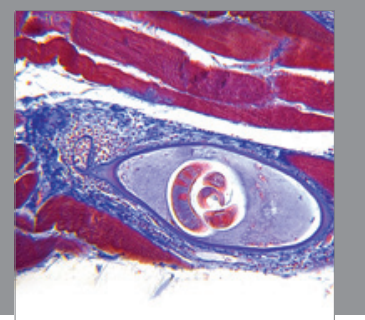

Gastroenterology

Research and Practice
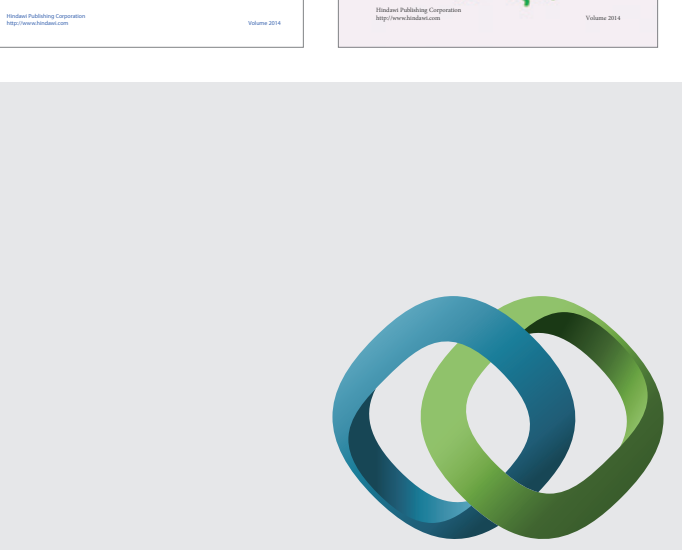

\section{Hindawi}

Submit your manuscripts at

http://www.hindawi.com
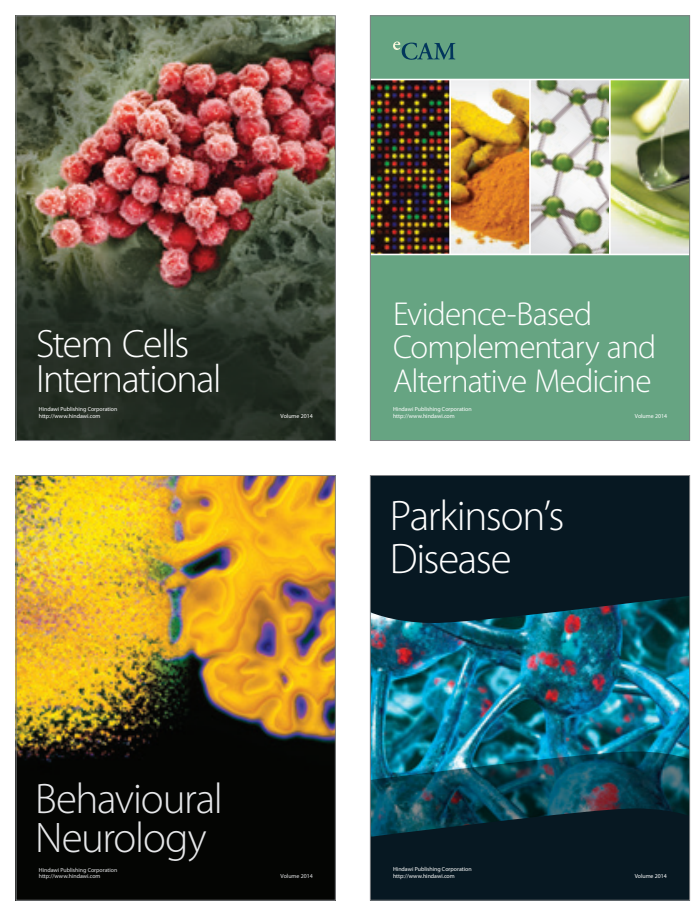

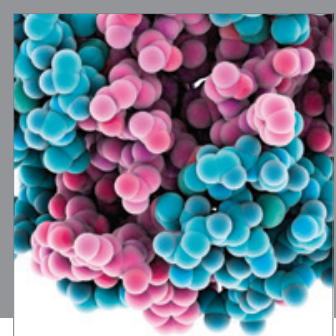

Journal of
Diabetes Research

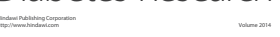

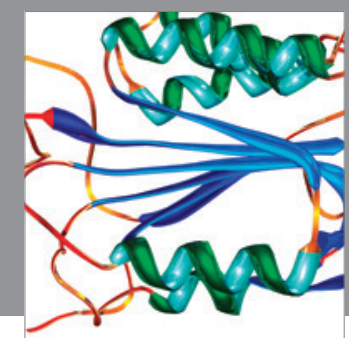

Disease Markers
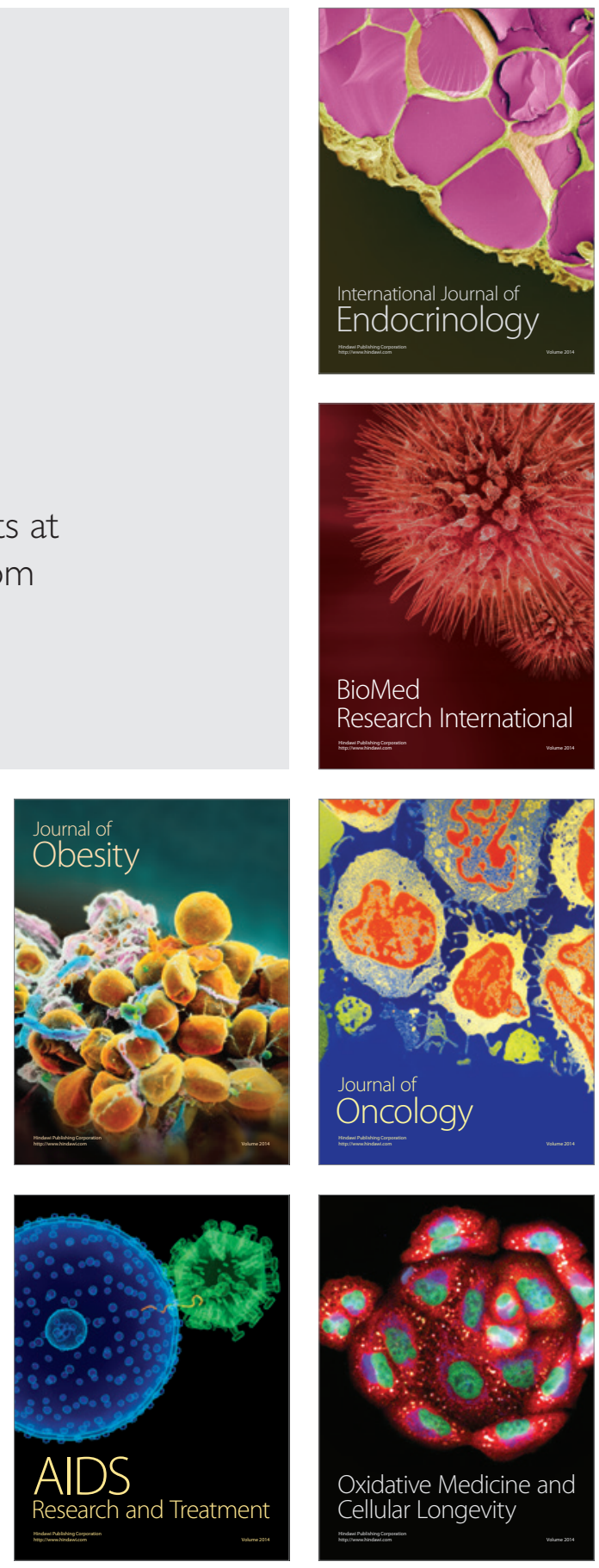